Brustkrebs:

\section{Personalisierte Behandlung soll Chemotherapie begrenzen}

Brustkrebs kann heute, wenn er frühzeitig entdeckt wird, in den meisten Fällen geheilt werden. Um eine Übertherapie zu vermeiden, suchen Mediziner nach Möglichkeiten, die Therapie auf das notwendige Maß zu begrenzen. Die personalisierte Therapie verspricht einen Weg dahin: Mit ihrer Hilfe soll aus einer länger werdenden Liste von Medikamenten das richtige Medikament für den richtigen Patienten zum richtigen Zeitpunkt ausgewählt werden. Dafür ist eine umfassende molekulare Charakterisierung des individuellen Tumors und der betroffenen Patientin erforderlich. Neue Hochdurchsatzverfahren erlauben schon heute, die molekulare Vielfalt des individuellen Brustkrebses besser abzubilden als je zuvor.

Neben der frühen Diagnose und der adäquaten Lokaltherapie mit Operation und zum Teil lokaler Bestrahlung sind die guten Behandlungsergebnisse beim Brustkrebs im Frühstadium vor allem der sogenannten (neo)adjuvanten, medikamentösen Therapie zu verdanken, berichtet Professor Dr. med. Andreas Schneeweiss, Vorstandsmitglied der Deutschen Gesellschaft für Senologie, Sektionsleiter Gynäkologische Onkologie am Nationalen Centrum für Tumorerkrankungen, Universitätsklinikum Heidelberg. Die neoadjuvante, medikamentöse Therapie beginnt schon vor der Operation und verfolgt das Ziel, bereits im Körper vorhandene Mikrometastasen zu vernichten. Die adjuvante Therapie schließt sich hingegen an die Operation an. Bisher erhielten alle Frauen im sel- ben Brustkrebsstadium und ähnlichen Risikofaktoren die gleiche Therapie. «Nach heutigem Kenntnisstand hat dies häufig eine Übertherapie oder Fehlbehandlung zur Folge», sagt Schneeweiss: «Einige Patientinnen würden auch mit weniger Medikamenten zum Beispiel ganz ohne Chemotherapie überleben.»

Eine bessere Unterscheidung gelingt heute zunehmend durch die molekularbiologische Untersuchung der Tumorzellen. «Sogenannte Genexpressionsanalysen zeigen, welche Gene in den Tumorzellen aktiv sind», erläutert Prof. Schneeweiss. «Das Muster dieser Genaktivierung, die Gen-Signatur, erlaubt dann Rückschlüsse auf die Aggressivität der Tumorzellen.» Studien untersuchen derzeit, ob die sogenannte «Intrinsische Signatur», die «Amsterdam-Signatur», der «Genomic Grade Index» oder der «Recurrence Score» erkennen können, welchen Patientinnen künftig eine intensive (neo)adjuvante Chemotherapie erspart werden könnte. Schneeweiss hofft, dass es etwa $15-30 \%$ sein werden, ohne natürlich den Ergebnissen der laufenden Studien vorgreifen zu können.

«Es ist höchste Zeit, die one-size-fits-allStrategie bei der Brustkrebsbehandlung zu verlassen», meint der Experte. Bei der personalisierten Brustkrebstherapie kommen zunehmend Medikamente zum Einsatz, die nur bei manchen Patientinnen wirken, ohne dass dies immer durch Tests vorhersehbar wäre. Diese Medikamente könnten laut Prof. Schneeweiss am besten im Rahmen einer neoadjuvanten Therapie getestet, beziehungsweise nach $\mathrm{Zu}$ lassung eingesetzt werden. Dabei erhalten die Patientinnen die Medikamente vor der Operation. Regelmäßige Untersuchungen von Gewebeproben des Tumors könnten zeigen, ob die Therapie anschlägt. Im Idealfall sind während der Operation keine lebenden Tumorzellen mehr nachweisbar. «Wir wissen heute schon, dass das Zerstören aller Krebszellen in der Brust und den Achselhöhlen-Lymphknoten bei einigen Brustkrebsformen die Chance auf Heilung stark erhöht», berichtet Schneeweiss.

Die neuen Konzepte der Brustkrebsbehandlung sind sehr komplex. Ihre Umsetzung wird nach Einschätzung von Prof. Schneeweiss nur in hochspezialisierten Krebszentren möglich sein. «Ein wichtiger Schritt für die rasche Übertragung von neuen Erkenntnissen in die Behandlung war die Schaffung sogenannter Comprehensive Cancer Center nach amerikanischem Vorbild», sagt der Experte: «Dies sind Institutionen wie zum Beispiel das Nationale Centrum für Tumorerkrankungen in Heidelberg, in denen Grundlagenforscher und Kliniker unter einem Dach zusammenarbeiten.»

\section{Deutsche Gesellschaft für Senologie}

Weitere Informationen bei

Deutsche Gesellschaft für Senologie

Pressestelle

Annette Affhüppe

Dieselstraße 2, 50859 Köln

Tel. +49 2234 7011-584, Fax -6584

presse@senologie.org

www.senologie.org

\title{
PharmaTicker+++ PharmaTicker+++ PharmaTicker+++ PharmaTicker+++
}

Philips. Das University Medical Center Utrecht und Royal Philips Electronics beginnen mit einer klinischen Pilotstudie zur Bewertung einer neuen Behandlungsform von Brustkrebs auf der Grundlage von hochfokussiertem Ultraschall, der über die Magnetresonanzbildgebung geführt wird (MRHIFU). Für die Durchführung dieser Studie hat Philips ein speziell für die Forschung entwickeltes und für die Anatomie der weiblichen Brust optimiertes System gebaut. «Diese neue Pilotstudie zielt auf Patientinnen mit kleinen Brusttumoren $\mathrm{ab}$, die noch keine Metastasen gebildet haben.», sagt Dr. Maurice van den Bosch, interventioneller Radiologe und Leiter der Forschung am University Medical Center Utrecht.

Philips Deutschland $\mathrm{GmbH}$

Anke Ellingen

anke.ellingen@philips.com
First Sensor. Mit dem neuen Gamma-Sondensystem steht ab 2013 erstmals ein gewinkelter Detektor für den intra- und extraoperativen Nachweis radioaktiv markierter Wächterlymphknoten zur Verfügung. Bei der Wächterlymphknoten-Detektion gibt es Anwendungsfälle, in denen mit einer Gamma-Sonde in schwer zugänglichen Körperbereichen gemessen werden soll. «Diese Stellen können mit einem geraden Instrument oft nicht ideal erreicht werden. Viele Chirurgen wünschen sich deshalb eine abgewinkelte Sonde, um sozusagen ‘um die Ecke` messen zu können. Wir schließen diese Anwendungslücke nun durch unsere gewinkelte Gamma-Sonde», so Christian Güttler, Entwicklungsingenieur bei der First Sensor AG.

First Sensor AG

Simone Burkhardt

simone.burkhardt@first-sensor.com
AstraZeneca hat mit der Phase-III-Zulassungsstudie FALCON (Fulvestrant and AnastrozoLe COmpared in hormonal therapy Naïve advanced breast cancer) begonnen. An der globalen klinischen Studie sollen 450 postmenopausale Patientinnen mit Hormonrezeptor-positivem lokal fortgeschrittenem oder metastasiertem Mammakarzinom teilnehmen, die sich noch keiner Hormontherapie unterzogen haben. Ziel ist, die Wirksamkeit und Verträglichkeit von Fulvestrant (Faslodex ${ }^{\circledR}$ ) 500 mg im Vergleich zu Anastrozol $1 \mathrm{mg}$ in dieser Patientinnenpopulation zu untersuchen. Rekrutierung und Aufnahme von Patientinnen in die Studie haben bereits begonnen. Weitere Informationen zur FALCON-Studie unter: www.clinicaltrials.gov

AstraZeneca $\mathrm{GmbH}$

Florian Dieckmann

florian.dieckmann@astrazeneca.com 